Gut and Liver, Vol. 10, No. 4, July 2016, pp. 642-648

\title{
The Wire-Grasping Method as a New Technique for Forceps Biopsy of Biliary Strictures: A Prospective Randomized Controlled Study of Effectiveness
}

\author{
Yasunobu Yamashita ${ }^{1}$, Kazuki Ueda $^{1}$, Yuki Kawaji ${ }^{1}$, Takashi Tamura ${ }^{1}$, Masahiro Itonaga $^{1}$, Takeichi Yoshida ${ }^{1}$, Hiroki \\ Maeda ${ }^{1}$, Hirohito Magari ${ }^{2}$, Takao Maekita ${ }^{1}$, Mikitaka Iguchi ${ }^{1}$, Hideyuki Tamai ${ }^{1}$, Masao Ichinose ${ }^{1}$, and Jun Kato ${ }^{1}$ \\ ${ }^{1}$ Second Department of Internal Medicine, Wakayama Medical University, and ${ }^{2}$ Department of Internal Medicine, Nokami Kosei General \\ Hospital, Wakayama, Japan
}

Background/Aims: Transpapillary forceps biopsy is an effective diagnostic technique in patients with biliary stricture. This prospective study aimed to determine the usefulness of the wire-grasping method as a new technique for forceps biopsy. Methods: Consecutive patients with biliary stricture or irregularities of the bile duct wall were randomly allocated to either the direct or wire-grasping method group. In the wiregrasping method, forceps in the duodenum grasps a guidewire placed into the bile duct beforehand, and then, the forceps are pushed through the papilla without endoscopic sphincterotomy. In the direct method, forceps are directly pushed into the bile duct alongside a guide-wire. The primary endpoint was the success rate of obtaining specimens suitable for adequate pathological examination. Results: In total, 32 patients were enrolled, and 28 (14 in each group) were eligible for analysis. The success rate was significantly higher using the wire-grasping method than the direct method (100\% vs 50\%, $p=0.016$ ). Sensitivity and accuracy for the diagnosis of cancer were comparable in patients with the successful procurement of biopsy specimens between the two methods ( $91 \%$ vs $83 \%$ and $93 \%$ vs $86 \%$, respectively). Conclusions: The wire-grasping method is useful for diagnosing patients with biliary stricture or irregularities of the bile duct wall. (Gut Liver 2016;10:642-648)

Key Words: Biliary tract; Endoscopic transpapillary biopsy; Biliary strictures; Surgical instruments; Sphincterotomy

\section{INTRODUCTION}

Endoscopic procedures for pancreatobiliary regions have been well-established, and are necessary for evaluation and decompression of obstructive processes in pancreatobiliary disease. Because confirmation of malignant biliary strictures is best done by tissue diagnosis via histology, tissue sampling from the lesion in the bile duct through endoscopic procedures has also been available and is frequently performed. As methods to obtain cells or tissues for pathological diagnosis of bile duct lesions, exfoliative bile cytology, biliary brushing cytology, and biliary forceps biopsy have been reported to have sensitivities for malignancy detection ranging from $15 \%$ to $32 \%,{ }^{1-3}$ from $35 \%$ to $83 \%,^{4-6}$ and from $60 \%$ to $88 \%,{ }^{5,7-9}$ respectively.

Thus, transpapillary biopsy using forceps offers better diagnostic ability than exfoliative bile cytology and biliary brushing. In addition, forceps biopsy has the advantage of allowing collection of specimens large enough for histological evaluation including typing the differentiation of cancer, which enables selection of chemotherapeutic agents and prediction of prognosis. Moreover, using forceps permits investigation of the preoperative spread of tumor by step biopsy.

Endoscopic sphincterotomy (EST) has frequently been performed to allow easy insertion of forceps into the bile duct for biopsy. For example, Nishikawa et al. ${ }^{10}$ reported that EST was carried out in 52.5\% of patients with bile duct cancer to obtain specimens with forceps. However, complications can occur during EST such as pancreatitis, hemorrhage, and perforation. ${ }^{11}$ In addition, the risk of cholangitis by retrograde infection could increase after EST. ${ }^{12}$ Therefore, procedures without EST would be preferable for forceps biopsy. However, insertion of forceps through the papilla without EST is technically difficult.

Correspondence to: Yasunobu Yamashita

Second Department of Internal Medicine, Wakayama Medical University, 811-1, Kimiidera, Wakayama 641-0012, Japan

Tel: +81-73-4472300, Fax: +81-73-4453616, E-mail: yasunobu@wakayama-med.ac.jp

Received on May 21, 2015. Revised on August 5, 2015. Accepted on October 1, 2015. Published online March 30, 2016

pISSN 1976-2283 eISSN 2005-1212 http://dx.doi.org/10.5009/gnl15231

() This is an Open Access article distributed under the terms of the Creative Commons Attribution Non-Commercial License (http://creativecommons.org/licenses/by-nc/4.0) which permits unrestricted non-commercial use, distribution, and reproduction in any medium, provided the original work is properly cited. 
The lack of an optimally designed forceps for use in biliary biopsy is also a problem. Forceps designed with a large cup and a stiff shaft can take sufficient specimens but are difficult to insert through the papilla. On the other hand, those designed with a small cup and a flexible shaft can relatively easily be inserted into the bile duct, but it is difficult to obtain sufficient specimens for histological evaluation.

To improve these shortcomings, we recently developed the 'wire-grasping method,' a novel technique using a guide-wire without EST. This method is conducted as follows: under endoscopic guidance, (1) a guide-wire is inserted into the bile duct, and then, using forceps, the wire is grasped while in the duodenum; (2) the duodenoscope is angled up and advanced to bring the forceps close to the papilla; and (3) the forceps are inserted into the bile duct while still grasping the guide-wire.

To verify the usefulness of this novel technique, a prospective randomized controlled study to compare it to the conventional method was performed.

\section{MATERIALS AND METHODS}

\section{Patients}

This was a prospective randomized controlled study per- formed by enrolling inpatients at Wakayama Medical University Hospital who were scheduled to undergo bile duct biopsy through an endoscopic procedure. Eligible patients had a biliary stricture or irregularities of the bile duct wall by computed tomography (CT) or endoscopic ultrasonography and required a pathological diagnosis to differentiate malignant from benign lesions. The exclusion criteria were age 18 years or younger, pregnant women, surgically altered anatomy of the upper gastrointestinal (GI) or pancreatobiliary region, history of sphincterotomy, ongoing pancreatitis, tumor located at the intrahepatic duct, and tumor exposed from the ampulla of Vater. This study was approved by the ethics committee of Wakayama Medical University and informed consent was obtained from each patient.

\section{Study procedures}

Patients were randomly allocated to either the wire-grasping or the direct method before starting the procedure. A random number sequence generated by a central computer was used to perform 1:1 randomization.

Tissue biopsy from the bile duct was performed by fluoroscopy-guided retrograde biliary biopsy during endoscopic retrograde cholangiopancreatography (ERCP). ERCP was performed according to a standard technique under intravenous sedation
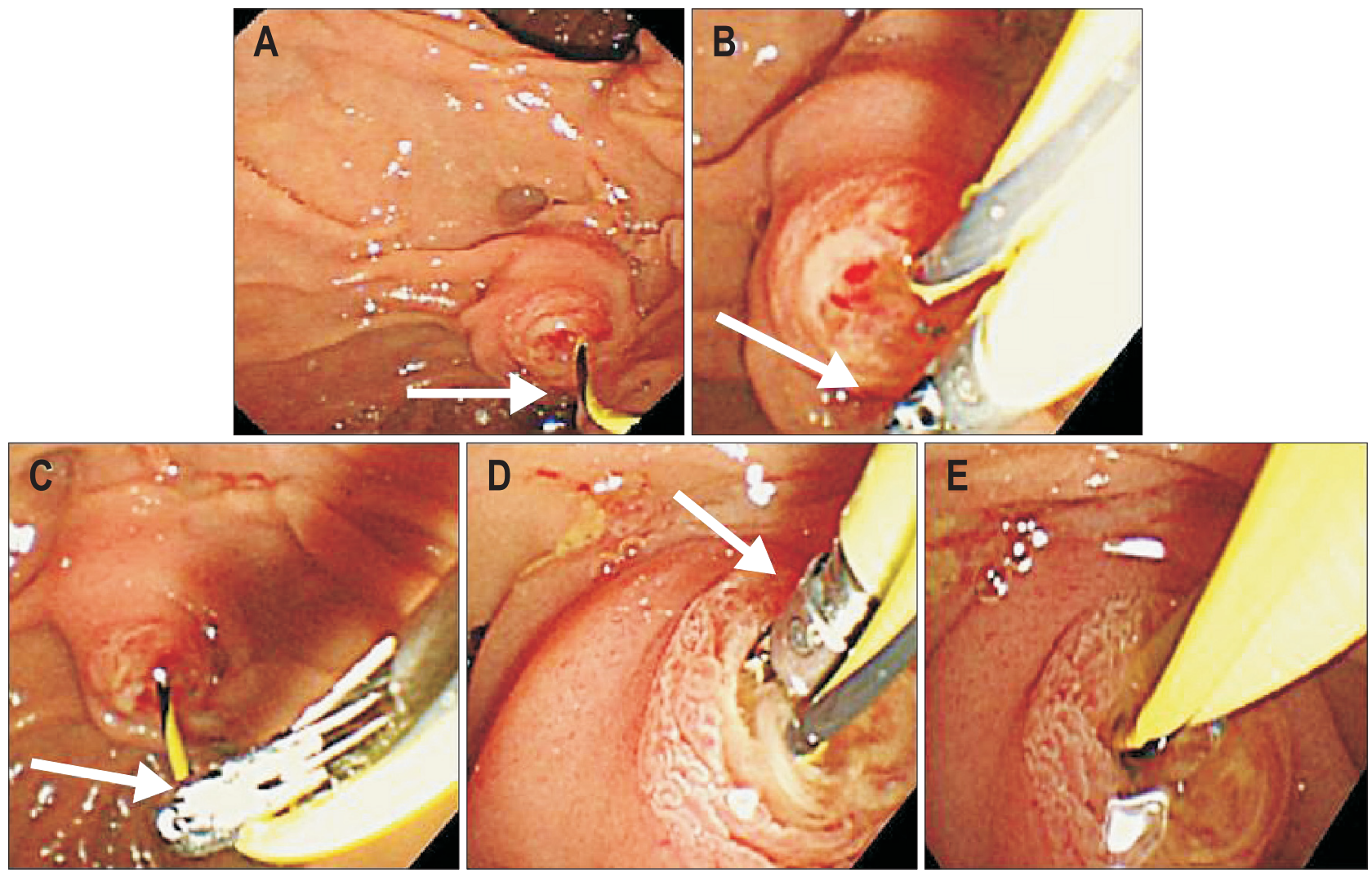

Fig. 1. The wire-grasping technique. (A) The warped guide-wire (arrow) is inserted into the bile duct through the papilla. (B) The forceps (arrow) are inserted through the endoscope channel. (C) The guide-wire is grasped by the forceps (arrow). (D) The duodenoscope is angled up and advanced to bring the wire-grasping forceps closer to the papilla (arrow). (E) The forceps are inserted into the bile duct while still grasping the guidewire. 
using the duodenoscope JF260V (Olympus, Tokyo, Japan) and the catheter RX ERCP Cannulas ${ }^{\circledR}$ (Boston Scientific, Natick, MA, USA). After successful selective cannulation, a guide-wire (0.035-inch Jagwire ${ }^{\circledR}$; Boston Scientific) was placed in the bile duct. In patients assigned to the wire-grasping method, the guide-wire was grasped in the duodenum by forceps inserted through the endoscope channel, which were then inserted through the papilla into the bile duct (Fig. 1). In patients assigned to the direct method, the forceps were directly inserted into the bile duct alongside the guide-wire. EST was not performed before forceps insertion into the bile duct in either group.

For biopsy, forceps with a 1.8-mm-diameter cup and 2.5- $\mathrm{mm}^{3}$ cup capacity (Radial Jaw $4 \mathrm{P}^{\circledR}$; Boston Scientific) were used. Radial Jaw $4 \mathrm{P}^{\circledR}$ is a disposable forceps mainly used for biopsy of the upper GI mucosa (esophagus, stomach, and duodenum) during upper GI endoscopy. These forceps have a neck swing function and reduced frictional resistance in the endoscope channel, so that they can easily take specimens from a tangential lesion; they are considered suitable for bile duct biopsy.

If cannulation with the forceps failed 5 times by using the allocated procedure, the other method was attempted. If both methods resulted in failure in forceps cannulation, the methods were repeated after performing EST. When forceps cannulation succeeded, two or more biopsy specimens were taken.

The biopsy specimens were fixed in 10\% formalin, and then embedded in paraffin. The histological diagnosis was made by examination from a board-certificated pathologist of hematoxylin and eosin stained slides. The histological diagnosis of cancer using biopsy specimens was validated by the final diagnosis determined by either examination of tissue specimens obtained during surgery or observation of the clinical course for more than 1 year in patients who did not undergo surgery.

The main outcome measure of this study was to determine the success rate of obtaining specimens suitable for adequate histopathological examinations. The secondary outcome measures were the following: to compare the times required for the two methods, defined as the period between the start of forceps insertion through the endoscopy channel and procurement of a biopsy sample; to assess complications associated with the methods; and to compare the methods for sensitivity, specificity and accuracy for diagnosis of cancer.

\section{Statistical analysis}

The required sample size was calculated by the results of our clinical experiences because of the absence of previous referential studies. The assumption of 90\% success rate for the wiregrasping method and 20\% for the direct method with an $\alpha$ error of 0.05 and a $\beta$ error of 0.10 elicited a sample size of 32 patients. To allow for problems of inappropriate enrollment or technical procedural problems, the target sample number was defined as 36 (18 in each group). The chi-square test or McNe- mar test was used for the statistical comparison of categorical variables between the two groups. The Mann-Whitney U test was used for the comparison of the procedural time period. A difference was considered significant when the $p$-value was less than 0.05. Statistical analysis was performed with SPSS version 11.0 (SPSS Inc., Chicago, IL, USA).

\section{RESULTS}

Between February 2013 and October 2014, 33 consecutive patients with biliary stricture or irregularities were considered eligible, but 1 was excluded due to Roux-en-Y reconstruction. A total of 32 patients (20 men, 12 women) were enrolled, and 16 were allocated to each method group (Fig. 2). Among these, four (two wire-grasping method group, two direct method group) were excluded from analysis because of failed insertion of the duodenoscope to the papilla due to duodenal invasion of cancer (one case) and failed placement of the guide-wire into the bile duct due to massive bile duct invasion of cancer (three cases). Enrollment was discontinued before reaching the predetermined number of patients because the superiority of the wire-grasping method group was demonstrated, and a total 28 patients were analyzed.

The demographic and disease characteristics of enrolled patients are shown in Table 1, and no significant differences between the groups were observed. The final diagnoses of these

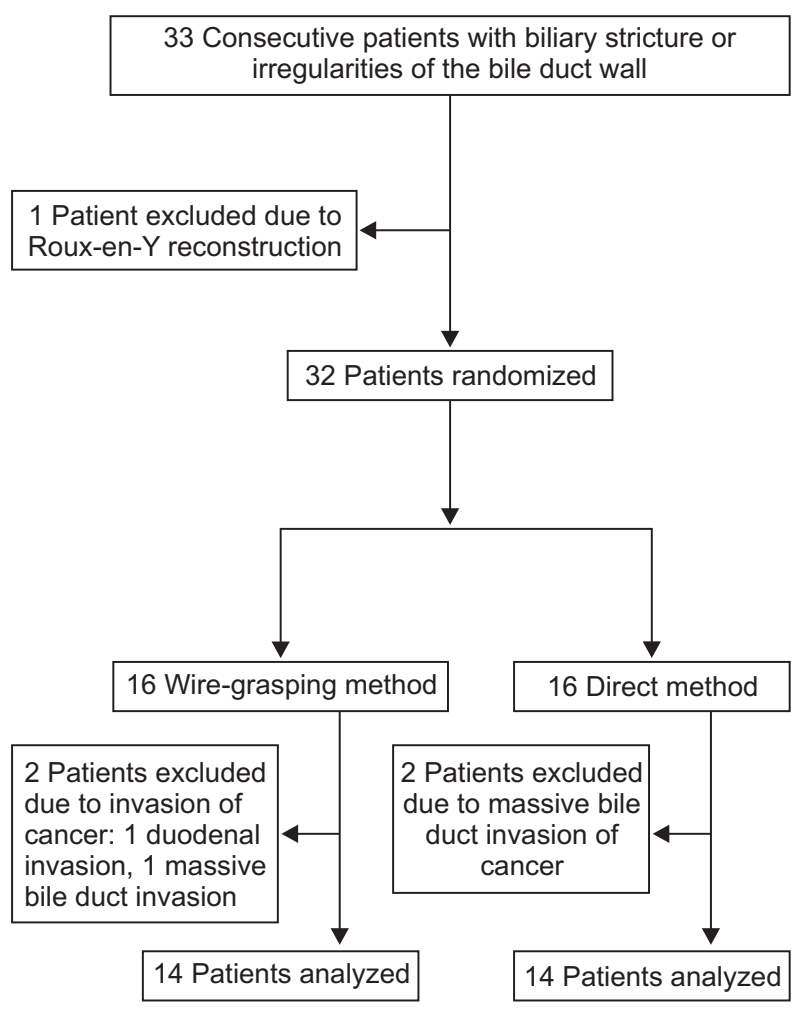

Fig. 2. Flowchart for patient enrollment. 
Table 1. Demographic and Disease Characteristics of the Two Study Groups

\begin{tabular}{lccc}
\multicolumn{1}{c}{ Characteristic } & Wire-grasping method (n=16) & Direct method (n=16) & p-value \\
\hline Age, yr & $75(63-85)$ & $74(46-87)$ & 0.78 \\
Male/female & $9 / 7$ & $11 / 5$ & 0.55 \\
Location (Bs, Bp/Bm/Bi) & $0 / 5 / 11$ & $2 / 3 / 11$ & 0.24 \\
Bs, Bp & 0 & 2 & 0.34 \\
Bm & 5 & 3 & 1 \\
Bi & 11 & 11 & 0.72 \\
Final diagnosis & & & 0.50 \\
Bile duct cancer & 8 & 1 & 0.36 \\
Bile duct cancer suspected & 0 & 6 & 1 \\
Pancreatic cancer & 4 & 1 & 0.30 \\
Cancer of the ampulla of Vater & 1 & 1 & \\
Benign stricture & 3 & & \\
\hline
\end{tabular}

Data are presented as median (range) or number.

$\mathrm{Bs}$, superior bile duct; $\mathrm{Bm}$, middle bile duct; $\mathrm{Bi}$, inferior bile duct; $\mathrm{Bp}$, portal bile duct.

Table 2. Results of the Use of the Two Methods

\begin{tabular}{lccc}
\hline \multicolumn{1}{c}{ Variable } & Wire-grasping method $(\mathrm{n}=14)$ & Direct method $(\mathrm{n}=14)$ & $\mathrm{p}$-value \\
\hline Success rate of obtaining adequate specimens & $14 / 14(100)$ & $7 / 14(50)$ & 0.016 \\
Sensitivity for cancer & $10 / 11(91)$ & $5 / 6(83)$ & 0.6 \\
Specificity for cancer & $3 / 3(100)$ & $1 / 1(100)$ & 1 \\
Accuracy for cancer & $13 / 14(93)$ & $6 / 7(86)$ & 0.57 \\
Time for procedure, min & $3.5(1-15)$ & $6(3-34)$ & 0.27 \\
Complications & $1 / 14(7)$ & $2 / 14(14)$ & 0.5 \\
\hline
\end{tabular}

Data are presented as number/total number (\%) or median (range).

patients were 15 bile duct cancers, one bile duct cancer suspected, six pancreatic cancers, two cancers of the ampulla of Vater, and four benign strictures. The patient whose final diagnosis was "suspected bile duct cancer" had shown typical features of bile duct cancer on contrast-enhanced CT, but could not be followed after the pathological diagnosis of biopsy specimens was determined to be atypical epithelium.

Successful cannulation of the forceps and successful procurement of biopsy specimens were achieved in 14 of 14 (100\%) of the wire-grasping method group. In contrast, the forceps could not be inserted in 7 of 14 of the patients (50\%) in the direct method group, and therefore, biopsy specimens could not be obtained in these patients. Consequently, the success rate of obtaining adequate specimens for pathological examinations was significantly higher in the wire-grasping method group than in the standard method group (100\% vs 50\%, respectively; $\mathrm{p}=0.016$ ). All seven patients for whom adequate specimens were not obtained by the direct method subsequently underwent the wire-grasping method, and successful biopsy was accomplished in six. The remaining patient required EST for collection of sufficient specimens.
There were no significant differences in sensitivity and accuracy for diagnosis of cancer in patients with successful procurement of biopsy specimens between the two groups. (91\% vs $83 \% ; p=0.6$, and $93 \%$ vs $86 \% ; p=0.57$, respectively). In addition, there was no significant difference between the two groups for the procedure time $(p=0.27)$. Regarding complications associated with the procedures, post-ERCP pancreatitis occurred in one patient of the wire-grasping method group and in two of the direct method group (7\% vs $14 \% ; p=0.5$ ) (Table 2). No other procedure-related severe complications occurred in either group.

\section{DISCUSSION}

Because malignant biliary lesions are sometimes difficult to distinguish from benign lesions by nonsurgical procedures, tissue sampling is highly desirable for precise diagnosis. A percutaneous transhepatic approach for obtaining tissue samples involves the risk of carcinomatous dissemination; hence, an endoscopic transpapillary approach is preferable to attain the purpose.

Among transpapillary methods, forceps biopsy has been 
shown to be superior to other techniques, including exfoliative bile cytology and biliary brushing cytology, ${ }^{13-15}$ for obtaining specimens. The technical feasibility of procuring biopsy specimens with forceps at ERCP was initially demonstrated in 1986. ${ }^{9}$ In 1995, Ponchon et al. ${ }^{4}$ reported in their study of a large number of patients that the tissue collection rate by means of forceps biopsy was 57\% (128/223). In the next year, Sugiyama et al. ${ }^{1}$ reported that tissue samples could be collected by forceps biopsy in $87 \%$ of patients (45/52). The failures of forceps biopsy could be attributed to difficulties with insertion of forceps into the bile duct and with firmly grasping tissues of lesions having irregular angles. Therefore, EST prior to forceps insertion and improvement of the forceps devices have been considered promising to ameliorate unsatisfactory situations.

EST can broaden the entry of the bile duct and could be expected to make insertion of the forceps easier. In fact, the procedure was sometimes performed during transpapillary bile duct forceps biopsy in previous studies. ${ }^{5,10,14,16}$ However, although more than $50 \%$ of subjects of these studies underwent EST, collection of tissue samples was not always satisfactory, and the sensitivities for malignancy diagnosis ranged from 50\% to 64\%. Moreover, EST can cause complications, hemorrhage in particular. The rates of hemorrhage after EST have been reported to be $2 \%$ to $14 \% .^{11,17-19}$ Thus, performing EST for forceps biopsy is not always effective, and may increase complications, including hemorrhage. Moreover, biopsy without EST has another advantage that stent placement for malignant lesions could be achieved without EST. Recent studies have indicated advantages of non-EST stent placement for malignant biliary strictures over stenting after EST, ${ }^{20-23}$ and therefore, the biopsy method without EST would be preferred according to the recent trend.

As one of the approaches for more efficient forceps biopsy, Howell et al. ${ }^{13}$ developed a 10-F introducer-type device. The instrument is a 10-F double-lumen catheter with a 0.035-inch guide-wire channel and a 5-F forceps channel. The forceps can reach the lesion through the forceps channel of the device which is introduced beforehand to the bile duct over a guidewire. Tissue samples could be successfully obtained without EST in all 28 patients who underwent forceps biopsy with the device. However, the sensitivity of the pathological examination using the collected samples for correct clinical diagnosis was only $31 \% .{ }^{13}$ The low sensitivity could be attributed to the small diameter of the cup $(1.5 \mathrm{~mm})$ of the forceps used (FB-38W ${ }^{\circledR}$; Olympus America Inc., Melville, NY, USA) and the $30^{\circ}$ angle between the forceps channel and the wire channel. Moreover, it appeared to be difficult to press the forceps against the bile duct wall because the shaft of the forceps was not stiff enough.

In this regard, the standard forceps FB-39Q ${ }^{\circledR}$ (Olympus) with a $1.8 \mathrm{~mm}$-diameter cup, used in many recent studies, ${ }^{1,5,14,16,24}$ may not be the best for forceps biopsy of bile duct lesions, because its shaft is relatively flexible. In fact, one study reported the su-

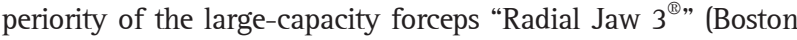
Scientific) in sensitivity for malignancy diagnosis over the standard forceps (70\% vs 43\%). ${ }^{16}$ The shaft of the "Radial Jaw $3^{\circledR}$ " is stiffer than that of the FB-39Q ${ }^{\circledR}$, and it can be pressed more efficiently against the bile duct wall. However, in the study, biopsy was performed with EST in all patients but one because the stiffness and the large cup size $(2.2 \mathrm{~mm})$ of Radial Jaw $3^{\circledR}$ made insertion through the papilla difficult.

Therefore, in our study, the next-generation, small-type Radial Jaw ${ }^{\circledR}$ forceps (Radial Jaw $4 \mathrm{P}^{\circledR}$ ) was used to perform procedures without EST. The cup diameter of the Radial Jaw $4 \mathrm{P}^{\circledR}$ (1.8 $\mathrm{mm}$ ) is smaller than that of Radial Jaw $3^{\circledR}$. However, Radial Jaw $4 \mathrm{P}^{\circledR}$ has the following advantages over the larger counterpart. First, the hard part at the distal end of the shaft is shorter, which makes insertion of the forceps through the papilla easier because it allows close proximity between the endoscope tip and papilla. Second, the open angle is wider $\left(150^{\circ}\right.$ vs $\left.120^{\circ}\right)$, enabling the operator to take larger samples even with the smaller cup. Third, Radial Jaw $4 \mathrm{P}^{\circledR}$ has a neck swing function and causes less frictional resistance in the endoscopy channel, which enables easier transmission of pressure through the shaft and easier procurement of specimens from a tangentially oriented lesion. Thus, the device is considered more suitable for bile duct biopsy. In fact, although the cannulation rate of the forceps in the direct method was lower than the result in Lin et al.'s study, ${ }^{25}$ our study was not inferior in terms of sensitivity and accuracy for cancer ( $83 \%$ vs $60 \%$ and $86 \%$ vs $67 \%$, respectively). The difference in cannulation rate might be attributable to the stiffness and the large cup size (1.8 $\mathrm{mm}$ vs $1.5 \mathrm{~mm}$ ) of the forceps we used in this study. In this regard, lower cannulation rate could be retrieved in part by using the wire-grasping method.

The most relevant advantages of the wire-grasping method demonstrated in the current study are the high successful cannulation rate and high sensitivity for pathological diagnosis of cancer. It should also be highlighted that these excellent results were obtained without performing EST. Moreover, there are several additional advantages to this method. First, it can be performed without any special devices, which means that the procedure is practical with low cost. Second, the method harbors no risk of accidental cannulation into the pancreatic duct. In contrast, there is a risk of accidental insertion of biopsy forceps into the pancreatic duct using the direct method, although this did not occur in our study. This fact is quite relevant because forceps are sometimes inserted repeatedly, particularly to perform step biopsy for the purpose of determining the required surgical margin. Moreover, in this situation, grasping a wire has another advantage in that the forceps can reach more distally when passing through a duct narrowed by tumor. Thus, the wire-grasping method appears to be safer and more useful than the direct method.

Our study had limitations. First of all, the number of enrolled 
patients was small and the study was performed in a single center. Although the wire-grasping method proved to be superior to the direct method in this small number of cases, the sample size seems to be too small to evaluate the complications associated with the procedures, including pancreatitis and perforation. Before generalizing our results, studies in other institutions and by other endoscopists should be performed. In addition, the unblinded nature of the study, which is inevitable in this type of study, might have influenced the outcome measures.

In conclusion, we established a novel, easier, and more secure method for performing forceps biopsy of bile duct lesions. We expect that this technique will facilitate more precise diagnosis of patients with biliary stricture or irregularities of the bile duct wall.

\section{CONFLICTS OF INTEREST}

No potential conflict of interest relevant to this article was reported.

\section{REFERENCES}

1. Sugiyama M, Atomi Y, Wada N, Kuroda A, Muto T. Endoscopic transpapillary bile duct biopsy without sphincterotomy for diagnosing biliary strictures: a prospective comparative study with bile and brush cytology. Am J Gastroenterol 1996;91:465-467.

2. Davidson B, Varsamidakis N, Dooley J, et al. Value of exfoliative cytology for investigating bile duct strictures. Gut 1992;33:14081411.

3. Mansfield JC, Griffin SM, Wadehra V, Matthewson K. A prospective evaluation of cytology from biliary strictures. Gut 1997;40: 671-677.

4. Ponchon T, Gagnon P, Berger F, et al. Value of endobiliary brush cytology and biopsies for the diagnosis of malignant bile duct stenosis: results of a prospective study. Gastrointest Endosc 1995;42: 565-572.

5. Pugliese V, Conio M, Nicolo G, Saccomanno S, Gatteschi B. Endoscopic retrograde forceps biopsy and brush cytology of biliary strictures: a prospective study. Gastrointest Endosc 1995;42:520526.

6. Cohen MB, Wittchow RJ, Johlin FC, Bottles K, Raab SS. Brush cytology of the extrahepatic biliary tract: comparison of cytologic features of adenocarcinoma and benign biliary strictures. Mod Pathol 1995;8:498-502.

7. Rustgi AK, Kelsey PB, Guelrud M, Saini S, Schapiro RH. Malignant tumors of the bile ducts: diagnosis by biopsy during endoscopic cannulation. Gastrointest Endosc 1989;35:248-251.

8. Kubota Y, Takaoka M, Tani K, et al. Endoscopic transpapillary biopsy for diagnosis of patients with pancreaticobiliary ductal strictures. Am J Gastroenterol 1993;88:1700-1704.

9. Aabakken L, Kåresen R, Serck-Hanssen A, Osnes M. Transpapillary biopsies and brush cytology from the common bile duct. Endoscopy 1986;18:49-51.

10. Nishikawa T, Tsuyuguchi T, Sakai Y, et al. Factors affecting the accuracy of endoscopic transpapillary sampling methods for bile duct cancer. Dig Endosc 2014;26:276-281.

11. Freeman ML, Nelson DB, Sherman S, et al. Complications of endoscopic biliary sphincterotomy. N Engl J Med 1996;335:909-918.

12. Yasuda I, Tomita E, Enya M, Kato T, Moriwaki H. Can endoscopic papillary balloon dilation really preserve sphincter of Oddi function? Gut 2001;49:686-691.

13. Howell DA, Parsons WG, Jones MA, Bosco JJ, Hanson BL. Complete tissue sampling of biliary strictures at ERCP using a new device. Gastrointest Endosc 1996;43:498-502.

14. Schoefl R, Haefner M, Wrba F, et al. Forceps biopsy and brush cytology during endoscopic retrograde cholangiopancreatography for the diagnosis of biliary stenoses. Scand J Gastroenterol 1997; 32:363-368.

15. de Bellis M, Sherman S, Fogel EL, et al. Tissue sampling at ERCP in suspected malignant biliary strictures (Part 2). Gastrointest Endosc 2002;56:720-730.

16. Ikeda M, Maetani I, Terada K, et al. Usefulness of endoscopic retrograde biliary biopsy using large-capacity forceps for extrahepatic biliary strictures: a prospective randomized study. Endoscopy 2010;42:837-841.

17. Jin PP, Cheng JF, Liu D, Mei M, Xu ZQ, Sun LM. Endoscopic papillary large balloon dilation vs endoscopic sphincterotomy for retrieval of common bile duct stones: a meta-analysis. World J Gastroenterol 2014;20:5548-5556.

18. Tanaka Y, Sato K, Tsuchida H, et al. A prospective randomized controlled study of endoscopic sphincterotomy with the Endocut mode or conventional blended cut mode. J Clin Gastroenterol 2015;49:127-131.

19. Kim KY, Han J, Kim HG, et al. Late complications and stone recurrence rates after bile duct stone removal by endoscopic sphincterotomy and large balloon dilation are similar to those after endoscopic sphincterotomy alone. Clin Endosc 2013;46:637-642.

20. Wilcox CM, Kim H, Ramesh J, Trevino J, Varadarajulu S. Biliary sphincterotomy is not required for bile duct stent placement. Dig Endosc 2014;26:87-92.

21. Artifon EL, Sakai P, Ishioka S, et al. Endoscopic sphincterotomy before deployment of covered metal stent is associated with greater complication rate: a prospective randomized control trial. J Clin Gastroenterol 2008;42:815-819.

22. Cui PJ, Yao J, Zhao YJ, Han HZ, Yang J. Biliary stenting with or without sphincterotomy for malignant biliary obstruction: a metaanalysis. World J Gastroenterol 2014;20:14033-14039.

23. Zhou H, Li L, Zhu F, Luo SZ, Cai XB, Wan XJ. Endoscopic sphincterotomy associated cholangitis in patients receiving proximal biliary self-expanding metal stents. Hepatobiliary Pancreat Dis Int 2012;11:643-649.

24. Higashizawa T, Tamada K, Tomiyama T, et al. Biliary guidewire 
648 Gut and Liver, Vol. 10, No. 4, July 2016

facilitates bile duct biopsy and endoscopic drainage. J Gastroenterol Hepatol 2002;17:332-336.

25. Lin LF, Siauw CP, Ho KS, Tung JN. Guidewire technique for endoscopic transpapillary procurement of bile duct biopsy specimens without endoscopic sphincterotomy. Gastrointest Endosc 2003;58:272-274. 